\title{
Pneumologen setzen auf Phytotherapeutikum
}

- Nach der aktuellen Leitlinie der Deutschen Gesellschaft für Pneumologie gibt es kaum Präparate gegen akuten Husten, deren Wirksamkeit nach den Kriterien der evidenzbasierten Medizin nachgewiesen wurde. Eine Ausnahme bildet das Thymian-Efeu- bzw. Thymian-Primel-Kombinationspräparat, das aufgrund von zwei placebokontrollierten klinischen Studien für diese Indikation ausdrücklich empfohlen wird.

In den beiden randomisierten Doppelblindstudien wurde das Hustenmedikament Bronchipret ${ }^{\circledast}$ in den Darreichungsformen Saft (Thymian/Efeu) und Filmtabletten (Thymian/Primel) an 363 bzw. 362 erwachsenen Patienten mit akuter Bronchitis und produktivem Husten untersucht. Primärer Endpunkt war die Reduktion der täglichen Hustenanfälle im Mittel der Behandlungstage sieben bis neun gegenüber dem Ausgangspunkt. In beiden
Studien erwiesen sich die Darreichungsformen als ähnlich gut wirksam und reduzierten die Hustenfrequenz im Vergleich zu

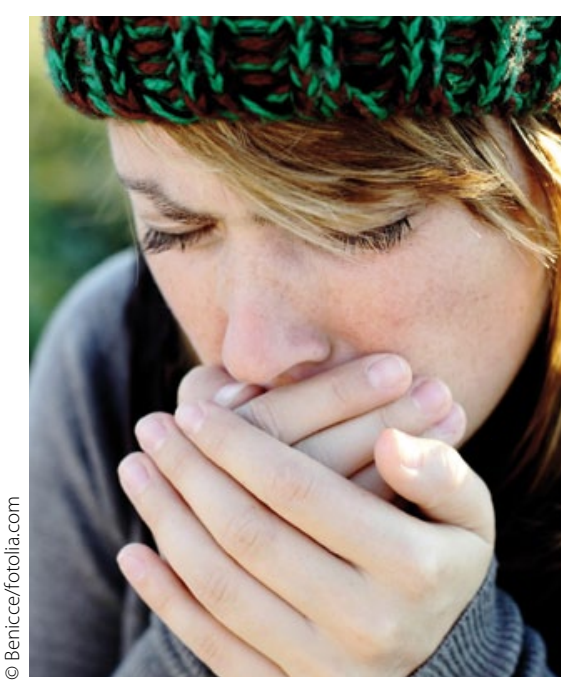

Thymian kombiniert mit Efeu oder Primel reduzierte die Zahl der Hustenanfälle.
Placebo signifikant: in der Verumgruppe mit dem Thymian-Efeu-Saft verringerte sich die Anzahl der Hustenattacken um 68,7\% (Placebo: 47,6\%), unter den Thymian-Primel-Tabletten um 67,1\% (Placebo: 51,3\%). Die Zahl der Hustenanfälle halbierte sich in beiden Studien bei der Hälfte der Patienten um zwei Tage schneller mit dem Phytotherapeutikum als unter Placebo.

Auch hinsichtlich weiterer Zielparameter wie z. B. Abhusten tagsüber, hustenbedingte Schlafstörungen oder allgemeines Wohlbefinden war das Prüfpräparat überlegen. Die Verträglichkeit wurde von Patienten und Prüfärzten in beiden Studien in 95-100\% der Fälle als "gut" bis „sehr gut" bewertet. Es kam zu keinen schwerwiegenden unerwünschten Ereignissen.

- Red

Nach Angaben von Bionorica SE

\section{Strukturierte Blutzuckerselbstmessung}

\section{Speziell geschulte Ärzte können den Nutzen weiter verbessern}

- Diabetiker profitieren von einer strukturierten Blutzuckerselbstmessung (BZSM), wenn die Werte zu einer angemessenen Verhaltens- und Therapieänderung führen, wie die Studie STeP ergab (Polonsky WH et al. Diabetes Care 2011; 34: 262-7). Noch effektiver könnte dies sein, wenn es den Ärzten durch eine entsprechende Schulung und ein Entscheidungshilfesystem erleichtert wird, ungünstige Blutzuckersituationen zu erkennen und die richtigen Therapieschritte einzuleiten, so das Ergebnis der Studie Accu-Chek ${ }^{\circledR}$ DECIDE.

In der prospektiven, kontrollierten Multicenterstudie wurden 288 Hausärzte am Computer mit 30 typischen, aber fiktiven Diabeteskasuistiken konfrontiert. In vier Gruppen eingeteilt, erhielten sie unterschiedliche Hilfsmittel. Die erste Gruppe (A) erhielt nur die visuelle Aufbereitung eines 7-Punkte-BZ-Profils über drei Tage, erfasst im Accu-Chek ${ }^{\circledR} 360^{\circ}$ View Tool. Die zweite Gruppe (B) erhielt zusätzlich die Berichte eines speziell entwickelten Entscheidungshilfesystems, das entsprechend der Werte Vorschläge zum weiteren Vorgehen macht. Die dritte Gruppe (C) erhielt stattdessen zusätzlich eine ca. 30-minütige Schulungs-DVD. Die vierte Gruppe D erhielt sowohl die DVD als auch die Berichte des Entscheidungshilfesystems. Die Teilnehmer sollten in den Fallbeispielen die dominierenden BZ-Abweichungen, etwa Hypoglykämie oder postprandiale Hyperglykämie, erkennen und adäquate Konsequenzen anbieten.

\section{Je mehr Hilfsmittel, desto besser}

Von den 233 Ärzten, die das Studienprotokoll erfüllt hatten, schnitt die Gruppe D mit allen drei Hilfsmitteln am besten ab. Signifikant mehr Ärzte (87\%) erkannten hier die primären Blutzuckerabweichungen als in den Gruppen A $(51 \%)$, B $(77 \%$,$) und C$ (72\%). Bei der richtigen Wahl der adäquaten Therapie lagen zwar die Ergebnisse der Gruppen B bis D dichter beieinander, waren aber alle signifikant besser als die der Gruppe A. Zu den Hilfsmitteln befragt gaben 90 bzw. 95\% der Teilnehmer an, vom Entscheidungshilfesystem und/oder von der DVD zusätzlich zum $360^{\circ}$ View Tool wesentlich profitiert zu haben.

Die Studienautoren schlossen daraus, dass der Fortschritt einer strukturierten BZSM durch ein Training der behandelnden Ärzte in der Interpretation und Anwendung dieses Hilfsmittels noch deutlich verbessert werden kann.

- Sarah L. Pampel

Quelle: Fachpressegespräch bei der EASD-

Jahrestagung, Lissabon, September 2011 (Veranstalter: Roche Diabetes (are) 